\title{
FS-EX Plus: A New Algorithm for the Calculation of Typical FS-Testor Set
}

\author{
Luis Roberto Morales-Manilla and Guillermo Sanchez-Diaz
}

Center of Technologies Research on Information and Systems, UAEH, Carr. Pachuca-Tulancingo Km. 4.5, C.P. 42084, Pachuca, Hgo., Mexico

luisroberto@lycos.com, sanchezg@uaeh.reduaeh.mx

\begin{abstract}
In this paper a novel exterior escale algorithm for the calculation of FS-typical testor set of a learning matrix is proposed. This algorithm allows to use any given similarity function between objects. Besides, results of experiments done, shows the performance obtained by proposed algorithm. A comparison between proposed algorithm and an exhaustive searching algorithm, that is the only one reported on literature that can calculate the complete FS-typical testor set, is also included.
\end{abstract}

Keywords: FS-testor, feature selection, pattern recognition, typical testor.

\section{Introduction}

A relevant task in supervised classification is feature selection. This task allows identifying those features that provide relevant information on the classification process. Into the framework of the Logical Combinatorial Pattern Recognition [1] and [2, feature selection is solved using Testor Theory [3]. Yu. I. Zhuravlev introduced the testor concept to pattern recognition problems 4. Zhuravlev defines a testor like a feature subset that does not confuse objects descriptions which belong to different classes. Subsequently, this concept has extended and generalized to adjust it in other ways 3. Another generalization model into testor theory is the FS-testors, developed by [5]. This model allows handle any similarity function among objects, opposed as classical testor models. This concept has a special application when qualitative and quantitative features are present (mixed data), using any similarity function among objects. Since computing all typical testors is very expensive, all developed algorithms have exponential complexity. Into the FS-testor model, the run time complexity depends of the similarity function handled. If the similarity function is very complex, then more run time of execution will necessary.

Currently, an exhaustive searching method is the only way in order to calculate the set of FS-typical testors. All feature combinations must be evaluated to determine if FS-testor property is fulfilled. The other way, consists in not to verify this property for every combination, carrying out "skips" of feature combinations over power feature set, we know a priori that these combinations do not generate any FS-typical testor. 
In this paper, we introduce an algorithm that performs "skips" over power feature set, eliminating a considerable calculations amount.

\section{Some Basic Concepts}

Let LM be a learning matrix composed by a set of $m$ objects $\Omega=\left\{O_{1}, O_{2}, \ldots, O_{m}\right\}$, described in terms of a set $R=\left\{X_{1}, X_{2}, \ldots, X_{n}\right\}$, with $n$ features of any nature (qualitatives or mixed quantitatives), where every feature $X_{j}$, has associated an admissible values set $M_{j}$. These objects are grouped on $K$ classes. Every attribute $X_{j}$ has associated a comparison criterion for the values of $X_{j}$, as a function in the form $C_{j}: M_{j} x M_{j} \rightarrow L_{j}$, where $L_{j}$ it is a totally ordered set.

Let $T$ be a feature combination such that $T \subseteq R$. Besides, let $R^{*} \subseteq R$ be a reference set. The following definitions were taken from [5, 6, 7].

Definition 1. The set $T \subseteq R$, is an FS-Differentiating feature set with respect to $\nu, D^{\prime}, R^{*}$ and $\beta$ of $L M$ if

$$
\begin{gathered}
\forall O_{i}, O_{j} \in L M\left[\nu\left(\bar{\alpha}\left(O_{i}\right), \bar{\alpha}\left(O_{j}\right)\right) \notin D^{\prime}\right] \Rightarrow \\
{\left[\beta\left(I_{/ T}\left(O_{i}\right), I_{/ T}\left(O_{j}\right)\right) \preceq \beta\left(I_{/ R *}\left(O_{i}\right), I_{/ R *}\left(O_{j}\right)\right)\right]}
\end{gathered}
$$

where $\bar{\alpha}\left(O_{j}\right)=\left(\bar{\alpha}_{1}\left(O_{j}\right), \ldots, \bar{\alpha}_{q}\left(O_{j}\right)\right)$ is the membership t-uple and $I_{/ T}\left(O_{p}\right)=$ $\left(x_{p 1}\left(O_{j}\right), \ldots, x_{p s}\left(O_{j}\right)\right)$ the subdescription or partial description of $O_{j}$ in terms of the features in $T$.

Definition 2. The set $T \subseteq R$, is an FS-Characteriziating feature set with respect to $\nu, D^{\prime}, R^{*}$ and $\beta$ of $L M$ if

$$
\begin{gathered}
\forall O_{i}, O_{j} \in L M\left[\nu\left(\bar{\alpha}\left(O_{i}\right), \bar{\alpha}\left(O_{j}\right)\right) \in D^{\prime}\right] \Rightarrow \\
{\left[\beta\left(I_{/ T}\left(O_{i}\right), I_{/ T}\left(O_{j}\right)\right) \succeq \beta\left(I_{/ R *}\left(O_{i}\right), I_{/ R *}\left(O_{j}\right)\right)\right]}
\end{gathered}
$$

Definition 3. The set $T \subseteq R$, is an FS-testor with respect to $\nu, D^{\prime}, R^{*}$ and $\beta$ of $L M$ iff $T$ is both FS-differentiating and FS-characteriziating set for $L M$, with respect to the same parameters.

In another hand, for the case of FS-typical testors, a partial order relation is required.

Let $\nu, D^{\prime}, \beta$ be given as parameters, and $L M, T_{1}, T_{2}$ and $R^{*}$ feature subsets of $R$, where $R$ is the total set of features of $L M$. The relation of partial order $\xi$ is defined as follows:

Definition 4. We say that $T_{1}$ antecedes to $T_{2}$ with respect to $\nu, D^{\prime}$, and $\beta$ in $L M$ and is denoted by $T_{1} \xi T_{2}$ iff satisfies a) or $\left.b\right)$ :

a) Exist a pair of objects $O_{k}, O_{l}$ of $L M$ such that $\beta\left(I_{/ T_{1}}\left(O_{k}\right), I_{/ T_{1}}\left(O_{l}\right)\right) \neq$ $\beta\left(I_{/ T_{2}}\left(O_{k}\right), I_{/ T_{2}}\left(O_{l}\right)\right)$, and $T_{1}$ is an FS-testor for the same parameters $\nu, D^{\prime}, T_{2}$ and $\beta$ of $L M$.

b) For every pair of objects $O_{i}, O_{j}$ of $M A$, $\beta\left(I_{/ T_{1}}\left(O_{k}\right), I_{/ T_{1}}\left(O_{l}\right)\right)=\beta\left(I_{/ T_{2}}\left(O_{k}\right), I_{/ T_{2}}\left(O_{l}\right)\right)$ and $T_{1} \xi_{2} T_{2}$ where $\xi_{2}$ is the following order relation defined in [8]: 


\section{$T 1 \xi_{2} T 2 \Leftrightarrow$}

$\left(T_{1} \cap T_{2}\right) \cup\left(\left(\right.\right.$ support $T_{1} \backslash$ support $\left.\left.T_{2}\right) \cap T 1\right) \cup\left(\left(\right.\right.$ support $T_{2} \backslash$ support $\left.\left.T_{1}\right) \cap T_{2}\right)=T_{2}$

Note that b) is intended for the case where the classes are fuzzy; however, in the case where classes are crisp, the result of this relation is the inclusion of sets.

Taking as base the ralation of parcial order before mentioned, we can give the next definition of FS-typical testor:

Definition 5. The set $T \subseteq R$, is an FS-typical testor with respect to $\nu, D^{\prime}, R *$ and $\beta$ of $L M$, if $T$ is an FS-testor for the same parameters and there are not $T^{\prime}, T^{\prime} \xi T$ that is an FS-testor with respect to $\nu, D^{\prime}, R *$ and $\beta$ of $L M$.

FS-EX Plus algorithm, in order to fulfill the "skips" over the power feature set, uses the same search order that 9]. This search order was chosen because it allows to the algorithm carry out the big amount of "skips", and these was larger possible.

The algorithm proposed, use the following premises in order to carry out the "skips".

Proposition 1. Let $T \subseteq R$ be a FS-testor. If $T^{\prime} \subseteq R, T \subseteq T^{\prime}$ then $T^{\prime}$ is not a FS-typical testor.

Proof. As $T \subseteq T^{\prime}$ (e.g. these are comparable sets) then $T$ antecedes $T^{\prime}$ by definition 4 (because only it is contemplated inclusion for crisp case). If $T^{\prime}$ was FS-testor, then by definition 5 of FS-typical testor, $T^{\prime}$ it is not an FS-typical testor.

Now, if $T^{\prime}$ was not an FS-testor, then by definition 5 of FS-typical testor, $T^{\prime}$ can not be neither an FS-typical testor.

Therefore, $T \subseteq T^{\prime}$ is not an FS-typical testor.

Proposition 2. Let $T \subseteq R, T^{\prime} \subseteq R$, such that $T \nsubseteq T^{\prime}$ and $T^{\prime} \nsubseteq T$ (e.g. these are incomparable sets). If $T$ is an FS-testor with respect to $\nu, D^{\prime}, T^{\prime}$ and $\beta, T$ is not an FS-typical testor.

Proof. As $T$ is an FS-testor with respect to $T^{\prime}$, then by definition $4, T$ anteceds $T^{\prime}$. And by definition $5, T^{\prime}$ isn't an FS-typical testor.

Proposition 3. Let $T \subseteq R$ be an FS-testor. If $\nexists T^{\prime} \subseteq R$, such that $T^{\prime}$ is an FStestor with respect to $\nu, D^{\prime}, T$ and $\beta$, such that $T^{\prime} \subseteq T$; and besides $\nexists T^{\prime \prime} \subseteq R$, such that $T^{\prime \prime}$ is an FS-testor with respect to $\nu, D^{\prime}, T$ and $\beta$, with $T \nsubseteq T^{\prime \prime}$ and $T^{\prime \prime} \nsubseteq T$, then $T$ will be an FS-typical testor.

Proof. a) Suppose that $\exists T^{\prime} \subseteq R$ such that $T^{\prime} \subseteq T, T^{\prime}$ FS-testor. Then, by proposition 1, $T$ is not an FS-typical testor!

b) Suppose that $\exists T^{\prime \prime} \subseteq R$ such that $T^{\prime \prime} \nsubseteq T, T \nsubseteq T^{\prime \prime}$ and $T^{\prime \prime}$ is an FS-testor with respect to $\nu, D^{\prime}, T$ and $\beta$. Then, by proposition $2, T$ is not an FS-typical testor!

Therefore $T$ is an FS-typical testor. 


\section{FS-EX Plus Algorithm}

In general, FS-EX Plus algorithm works in the following way. The first combination is generated in the established order and it verifies if this combination is an FS-testor. If so occur, then the combination is saved on a candidates list of FStypical testors and then all of its consequents supersets in the established order are skipped (proposition 1). In candidates list, all supersets of the current combination are deleted. In other case, the next combination is generated according the established order. This process is repeated until all possible combinations of the power set were generated.

Once the candidates list for FS-typical testors has been obtained, combinations are evaluated in order to eliminate those that being incomparables, and are anteceded among them (proposition 2). One a time all these operations have been done, the final list containing FS-typical testor found (proposition 3) is saved.

\section{Description of the algorithm FS-EX plus}

Input: LM (learning matrix)

Output: FSTT (FS-typical testor sets calculated)

\section{Inizialitation.- $F S T T=\emptyset ; C o m b=\left\{X_{1}\right\}$}

2. Evaluation of candidates.- If last possible feature combination in the search order has been reached, then go to step 5 (e.g. Comb $\left.=\left\{X_{n}\right\}\right)$. On another case, the property of FS-testor is verified. If Comb was FS-testor, then Comb is saved in FSTT $(F S T T=F S T T \cup C o m b)$. If there are a superset of Comb in FSTT, then these supersets are eliminated of FSTT (proposition 1). If Comb was not FS-testor, go to step 4.

3. Skip of non FS-typical testors combinations.- Like Comb was FS-testor, then FS_comb $=C o m b$ and Comb $=$ Next_combination $(C o m b)$ while Comb is a superset of $F S_{\_} c o m b$. This process means that subsequently supersets of FS_comb are skipped (proposition 1) until Comb is a non superset of FS_comb. Go to step 2.

4. Selection of the new combination to analyze.- The following feature combination for verify is constructed based on before combination generated $\left(C o m b=N e x t \_c o m b i n a t i o n(C o m b)\right)$. Go to step 2.

5. Generation of FS-typical testor set.- The FS-typical testor set is generated. Each Combination $\mathrm{Comb}_{i} \in F S T T$ is compared with remaining combinations of FSTT (e.g. $C o m b_{j} \in F S T T, i \neq j$ ). If $C o m b_{i}$ is and FS-testor with respect to $C o m b_{j}$, then $C o m b_{j}$ is eliminated of $F S T T$ (proposition 2). The FStypical testor set is composed by the feature combinations of FSTT (proposition 3). The algorithm finish. 
Function Next ombination $(\mathrm{Comb})$ return the next feature combination of $C o m b$ in the established order of the feature power set.

\section{Evaluation}

In order of verify the performance of proposed algorithm, a comparison with an exhaustive search algorithm was fulfilled. The exhaustive search algorithm evaluates all possible combinations from the power feature set.

For our comparison, we handled execution times and the number of FS-typical testor obtained, it was used several learning matrices containing real data and that were taken from [10]. Matrices belong to four data bases: Zoo, Votes, Mushroom and Wine.

Values of parameters used in tests were:

$$
\nu\left(\bar{\alpha}\left(O_{i}\right), \bar{\alpha}\left(O_{j}\right)\right)=\left\{\begin{array}{l}
1, \text { if } \bar{\alpha}\left(O_{i}\right)=\bar{\alpha}\left(O_{j}\right) \\
0, \text { if } \bar{\alpha}\left(O_{i}\right) \neq \bar{\alpha}\left(O_{j}\right)
\end{array}\right.
$$

and $D^{\prime}=\{1\}, R^{*}=R$.

Table 1 , shows the results obtained after calculating the FS-typical testors to mentioned matrices taking as similarity function the matching function used in typical testors.

Notation used in the table 1 is as follows:

ECE: Combinations evaluated by exhaustive algorithm

ECFS: Combinations evaluated by FX-EX plus algorithm

TE: Time in seconds used by exhaustive algorithm

TFS: Time in seconds used by FX-EX plus algorithm

NFSTT: Number of FS-typical testors generated

Table 1. Experimental results obtained to Zoo, Votes, Mushroom and Wine data sets

\begin{tabular}{|l|c|c|c|c|c|}
\hline Data set (rows x columns) & ECE & ECFS & TE & TFS & NFSTT \\
\hline Zoo (101x17) & 131071 & 22315 & 2145 & 96 & 53 \\
\hline Votes (435x16) & 65535 & 65534 & 1349 & 1342 & 3 \\
\hline Mushroom (8124x22) & 4194303 & 22 & $>86400$ & 1349 & 22 \\
\hline Wine (178x13) & 8191 & 146 & 191 & 2 & 67 \\
\hline
\end{tabular}

As we can observe in this results, FS-EX Plus algorithm complies with task of realize a significant less number of evaluations, in contrast with exhaustive search algorithm. However, it could be given the case where for a given matrix, the only FS-testor existing given a similarity function, were the last feature combination, as a result, the number of evaluated combinations in both algorithms would be dramatically the same. But the cause for this would be the chosen similarity function again. This allows to make emphasis in the complexity that involves working with FS-testors model, since in order to such combinations exist inside 
Table 2. Time proportion for calculations between exhaustive search and FS-EX Plus algorithms

\begin{tabular}{cc}
\hline Data set & time \\
\hline Zoo & FS-EX plus 22 times more fast than Exhaustive algorithm \\
Mushroom & FS-EX plus 64 times more fast than Exhaustive algorithm \\
Wine & FS-EX plus 95 times more fast than Exhaustive algorithm \\
\hline
\end{tabular}

a learning matrix, it depends of many factors, which ones can be, the learning matrix itself, the similarity function $\beta$ that is using or even the reference set $R *$ itself who has not either be always the total set of attributes.

From the obtained results, one can observe that FS-EX Plus algorithm found the set of FS-typical testors in the following time proportions:

For the experiment with LM of Mushroom, the process was stopped after 24 hours and was estimated that every realized combination took 164 seconds being evaluated, having to process 4,194303 combinations of attributes. Predicting that exhaustive search algorithm will finish in a few more of 14 years approximately.

\section{Conclusions}

In this article a novel algorithm of exterior scale to calculate all the set of FStypical testors was introduced.

The shown performance in the experiments by algorithm was more efficient than exhaustive search algorithm due the "skips" realized over power feature set, avoiding to verify a considerable quantity of combinations that do realizes the exhaustive search algorithm.

Proposed algorithm opens a way for the construction of new exterior escale algorithms to find FS-typical testors. These new algorithms could incorpore some order in the attributes of the learning matrix, or other run over power set of attributes that allows it to decrease the number of combinations to be verified.

It is relevant to say that this algorithm is the first one reported in the literature that does not verify all possible combinations of attributes and generates the total set of FS-typical testors. Besides, currently we do not found a reported in the literature exhaustive search algorithm developed.

Acknowledgement. To PhD. Manuel Lazo Cortés for all the ideas given to this project.

\section{References}

[1] Ruiz-Shulcloper, J., Abidi, M.: Logical Combinatorial Pattern Recognition: A Review. Recent Research Developments in Pattern Recognition. In: Pandalai, S.G. (ed.) Recent Research Developments in Pattern Recognition, Transworld Research Networks, Kerala, India, pp. 133-176 (2002) 
[2] Martinez-Trinidad, J.F., Guzmán Arenas, A.: The Logical Combinatorial approach for pattern recognition an overview through selected Works. Pattern Recognition 34(4), 741-751 (2001)

[3] Lazo-Cortes, M., Ruiz-Shulcloper, J., Alba Cabrera, E.: An overview of the evolution of the concept of testor. Pattern Recognition 34(4), 753-762 (2001)

[4] Dmitriev, A.N., Zhuravlev, Y.I., Krendeliev, F.P.: About mathematical principles and phenomena classification. Diskretni Analiz. 7, 3-15 (1966)

[5] Alba-Cabrera, E.: Novel extensions about testor model for several kinds of similarity functions. Ph. D. thesis, University of Havana, Cuba (1998)

[6] Alba-Cabrera, E., Lazo-Cortés, M.: A Global Solution for the Utilization of Testors in Pattern Recognition Problems. In: Proc. TIARP 1998, pp. 7-22 (1998) (In Spanish)

[7] Alba-Cabrera, E.: A testor concept for any analogy function in a totally ordered set. Integration Journal, Colombia 14(2), 75-82 (1996)

[8] Lazo Cortes, M.: Models based in testor theory for feature selection and supervised classification with non classical objects descriptions. Ph. D. Thesis, UCLV, Cuba (1994)

[9] Santiesteban Alganza, Y., Pons Porrata, A.: LEX: a new algorithm for calculated typical testors. Mathematics Sciences Journal 21(1), 85-95 (2003) (In Spanish)

[10] Newman, D.J., Hettich, S., Blake, C.L., Merz, C.J.: UCI Repository of machine learning databases. Irvine, CA: University of California, Department of Information and Computer Science (1998), http://www.ics.uci.edu/ mlearn/ MLRepository.html 